\title{
Evolución de la aspiración laringo-traqueal en la disfagia orofaríngea secundaria a lesión cerebral traumática: cuantificación videofluoroscópica
}

\author{
R. Terré y F. Mearin \\ Unidad de Rehabilitación Funcional Digestiva. Institut Guttmann. Hospital de Neuro-rehabilitación. Instituto \\ Universitario adscrito a la UAB. Badalona, Barcelona
}

\section{RESUMEN}

Introducción: la alteración de la deglución en la fase inicial de un traumatismo craneoencefálico grave (TCE) es muy frecuente.

Objetivo: definir y cuantificar las alteraciones videofluoroscópicas en pacientes después de un TCE y evaluar la evolución de los pacientes con aspiraciones laringo-traqueales.

Método: se estudiaron de forma prospectiva 10 pacientes con TCE grave con sospecha clínica de aspiración, que fue confirmada mediante exploración videofluoroscópica (VDF). La VDF se repitió al mes, 3, 6 y 12 meses de evolución.

Resultados: la exploración clínica demostró alteración en los reflejos palatal y nauseoso en el 30\% de los pacientes, y tos durante la exploración en el 40\%. En la primera exploración VDF se observó: aumento del tiempo de tránsito oral (TTO) en el 70\% (media: 3,8 seg.; rango: 0,8-15 seg.) y alteración en el control lingual en el $60 \%$, con disfunción en el sello glosofaríngeo en el 20\%. El tiempo medio de tránsito faríngeo (TTF) fue de 0,72 seg. (rango: 0,34-1,50 seg.) y el tiempo de disparo del reflejo deglutorio (DRD) de 0,32 seg. (rango: 0,10-0,80 seg.). Al año de evolución sólo 3 pacientes aspiraban, siendo el TTO normal en 7 pacientes, el DRD en 9 y el TTF en todos. El 80\% seguía dieta oral exclusiva y el $20 \%$ combinaban alimentación oral y por sonda de gastrostomía.

Conclusión: la VDF permite confirmar y cuantificar las alteraciones de la deglución en los pacientes con TCE grave. Los hallazgos más frecuentes en la fase inicial de la evolución son el aumento del TTO y la alteración en el control lingual; las aspiraciones son muy frecuentes, siendo más de la mitad aspiradores silentes. Al año la mayoría de pacientes ha evolucionado favorablemente.

Palabras clave: Traumatismo cráneoencefálico. Deglución. Disfagia. Aspiración. Videofluoroscopia. Cuantificación.

\begin{abstract}
Introduction: swallowing impairments are frequent after severe traumatic brain injury (TBI).

Objective: to define and prospectively quantify the videofluoroscopic symptoms in patients after TBI, and to evaluate the evolution of patients with laryngotracheal aspiration.

Method: we studied 10 patients with TBI, and a clinical suspicion of aspiration confirmed by means of a videofluoroscopic exploration (VDF). VDF was repeated at 1, 3, 6, and 12 months thereafter.

Results: $30 \%$ of patients had an impaired gag reflex, and $40 \%$ cough during oral feeding. In the first VDF exploration the following was observed: increased oral transit time (OTT) in 70\% (average: $3.8 \mathrm{sec}$.; range: $0.8-15 \mathrm{sec}$.), altered lingual control in $60 \%$, and dysfunctional palatoglossal closure in $20 \%$. Mean pharyngeal transit time (PTT) was $0.72 \mathrm{sec}$ (range: 0.34-1.50 sec.), and time to swallowing reflex (TSR) was $0.32 \mathrm{sec}$. (range: $0.10-$ $0.80 \mathrm{sec}$.$) . After one year only 3$ patients had aspiration -with a normal OTT in 7 patients, a normal PTT in 9, and a normal TSR in all; $80 \%$ had an exclusively oral diet, and 20\% combined oral intake and gastrostomy feeding.

Conclusion: videofluoroscopic evaluation allows to confirm and quantite swallowing dysfunction in patients with severe TBI. Most frequent early findings included an increase in OTT and alterations in lingual control; aspirations were quite frequent, and more than half were silent. After one year the majority of patients showed a favorable outcome.
\end{abstract}

Key words: Traumatic brain injury. Deglutition. Dysphagia. Aspiration. Videofluoroscopy. Quantification.

Terré R, Mearin F. Evolución de la aspiración laringo-traqueal en la disfagia orofaríngea secundaria a lesión cerebral traumática: cuantificación videofluoroscópica. Rev Esp Enferm Dig 2007; 99: 7-12.

Recibido: 28-08-06.

Aceptado: 27-10-06.

Correspondencia: Rosa Terré. Unidad de Rehabilitación Funcional Digestiva. Instituto Guttmann. Camí de Can Ruti s/n. 08916 Barcelona. e-mail: metges@guttmann.com

\section{INTRODUCCIÓN}

Las alteraciones de la deglución son muy frecuentes en pacientes con traumatismo craneoencefálico grave (TCE) ingresados para rehabilitación, apareciendo en 
un $25-61 \%$ de los casos (1-4). Los mecanismos por los que se producen alteraciones en la deglución son múltiples, siendo las lesiones cerebrales asociadas al mismo las responsables de las alteraciones en la fisiología deglutoria, a las que se añadirán los déficits cognitivos y conductuales, que además dificultarán tanto el diagnóstico como el tratamiento (5). Hay que recordar también que los antecedentes de intubación orotraqueal y las cánulas de traqueostomía pueden ser causa de aspiración (6-9).

La mayoría de autores describen como disfunción más frecuente la alteración en la eficacia de la fase oral y el retraso en la respuesta faríngea, con el subsiguiente riesgo de aspiración (entrada de contenido alimenticio dentro de la vía aérea por debajo de las cuerdas vocales). En términos biomecánicos, las aspiraciones pueden producirse antes (durante la fase oral), durante, o después del disparo del reflejo deglutorio. La respuesta fisiológica a la aspiración es la tos refleja, pero en pacientes neurológicos existe un elevado porcentaje de aspiradores silentes (la aspiración no induce tos), que según la literatura oscila del 40 al 60\% (10-13). Lógicamente estos enfermos están expuestos a un elevado riesgo de neumonías por aspiración. No obstante, la disfunción de la deglución en pacientes con TCE presenta generalmente una buena evolución, de manera que entre el $75-94 \%$ recuperarán la capacidad de alimentarse por vía oral $(14,15)$.

En la práctica clínica existe una variabilidad importante en el diagnóstico y tratamiento de la disfagia en los pacientes con TCE. En muchas ocasiones el diagnóstico se realiza tan sólo mediante la exploración física y la observación externa de la deglución, pero la evaluación de las alteraciones biomecánicas precisará de estudios videofluoroscópicos y, en ocasiones, manométricos $(5,12)$. El estudio videofluoroscópico permite realizar un diagnóstico fisiopatológico de la disfunción de la deglución, cuantificando diferentes aspectos de la fisiología orofaríngea, con los que podremos efectuar un diagnóstico más preciso del problema deglutorio y, a su vez, indicar planes terapéuticos específicos.

La evaluación y tratamiento precoz proporcionarán importantes beneficios, eliminando potenciales complicaciones como la neumonía por aspiración y la desnutrición (16). Por tanto, el objetivo terapéutico debe ser conseguir una deglución segura y eficaz evitando que se desarrollen complicaciones nutricionales y respiratorias.

El objetivo de este estudio fue definir y cuantificar las alteraciones videofluoroscópicas en pacientes después de un TCE y evaluar la evolución de los pacientes con aspiraciones laringo-traqueales.

\section{PACIENTES Y MÉTODO}

Se efectuó un estudio prospectivo en 10 pacientes (6 hombres y 4 mujeres) ingresados con TCE grave [puntuación media en la escala de coma de Glasgow en el momento del accidente de 4 (rango: $3-8$ )], durante el periodo de diciembre-2004 a abril-2005 para tratamiento neurorehabilitador. La media de edad era de 34 años (rango: 1672 años) y el tiempo medio de evolución desde el TCE al ingreso en nuestro hospital de 2,5 meses (rango: 1-5 meses). Todos los pacientes tenían el antecedente de intubación orotraqueal y de haber sido portadores de cánula de traqueostomía. En todos ellos existía la sospecha clínica de aspiración laringo-traqueal que fue confirmada mediante exploración videofluoroscópica.

\section{Exploración clínica}

La exploración clínica de la deglución incluyó la evaluación de los reflejos velofaríngeos (reflejo velopalatino y nauseoso) y la administración de bolos de 3, 5, 10 y $15 \mathrm{ml}$ a las viscosidades pudin, néctar y líquido (espesando el agua con espesantes farmacológicos -Resource espesante ${ }^{\circledR}-$ ), valorando la aparición de tos y/o cambios en la calidad de la voz con la deglución. La exploración se inicia con bolos a la viscosidad pudin pasando a las viscosidades néctar y líquido si no se observaba alguna alteración en la seguridad de la deglución.

\section{Exploración videofluoroscópica}

La exploración fue practicada con bolos de Gastropgrafin $^{\circledR}$ de 3, 5, 10 y $15 \mathrm{ml}$, con el paciente en sedestación y en proyección lateral. Se recogió una secuencia completa de la deglución en un vídeo de alta resolución Kay Digital Swallowing Worksatation ${ }^{\circledR}$ (New Jersey, EE.UU.). Con esta exploración se definieron los parámetros videofluoroscópicos que evalúan la eficacia y seguridad de la deglución tanto para la fase oral como para la faríngea. La exploración se efectuó con bolos de volumen progresivo a la viscosidad pudin, continuándose posteriormente con las viscosidades néctar y líquido; la exploración se interrumpió si el paciente no era capaz de colaborar o se observaba aspiración.

En la fase oral se analizaron los siguientes parámetros que definen la eficacia: a) tiempo de tránsito oral (TTO). Es el periodo de tiempo desde el inicio de movimiento del bolo dentro de la cavidad oral hasta que la cabeza del bolo pasa el punto en que la mandíbula cruza la base de la lengua (en individuos normales oscila entre 1-1,25 seg.); b) control lingual. Su alteración se traduce en una incapacidad para formar el bolo y en su propulsión hacia la parte posterior de la boca y faringe. Los parámetros videofluoroscópicos para evaluar la seguridad en la fase oral incluyeron: a) disfunción del sello gloso-faríngeo. Si éste falla, parte o todo el bolo caerá prematuramente dentro de la faringe (antes del disparo del reflejo deglutorio), pudiendo ser aspirado; b) deglución fraccionada. Resulta cuando el paciente deglute el bolo en varias porciones (se considera normal en bolos de tamaño grande, superiores a $15 \mathrm{ml}$ ). En la fase faríngea los datos investigados para 
definir la eficacia de la deglución fueron: a) regurgitación nasofaríngea. Se produce como consecuencia de un sello velofaríngeo inadecuado o debido a una incapacidad del bolo para atravesar el EES, por lo que asciende a la nasofaringe; b) residuo en la cavidad faríngea después de la deglución o acúmulo de parte del bolo a nivel de los senos piriformes; y c) anomalías en la apertura del esfínter esofágico superior (EES). Se ven como defectos de repleción en la pared posterior de la faringe, y se denominan frecuentemente "barras del cricofaringeo". Los parámetros videofluoroscópicos para evaluar la seguridad en la fase faríngea incluyeron: a) disparo del reflejo deglutorio (DRD). Cuando la cabeza del bolo pasa el punto en que la mandíbula cruza la base de la lengua se dispara el reflejo deglutorio y se inicia la fase faríngea de la deglución que finaliza cuando la cola del bolo pasa a través del EES (se considera normal por debajo de 0,24 seg.); b) tiempo de tránsito faríngeo (TTF). Es el periodo de tiempo en el que la cabeza del bolo va desde la base de la lengua hasta que la cola del bolo pasa a través de la región cricofaríngea (en condiciones normales es inferior a $1 \mathrm{seg}$.); c) penetración/aspiración. La penetración se define como el paso de contenido del bolo al vestíbulo laríngeo por encima de las cuerdas vocales. Cuando el contenido alimenticio atraviesa las cuerdas vocales y entra a la vía aérea hablamos de aspiración. Además, se recogió el momento en que se produjo la aspiración (antes del disparo del reflejo deglutorio, durante la contracción faríngea o postdeglutoria). También se objetivó la aparición o no de tos durante la aspiración, considerándose aspiraciones silentes aquellas que no desencadenan tos refleja.

En los pacientes en los que se detectó aspiración la exploración videofluoroscópica se repitió al mes, 3 meses, 6 meses y un año, evaluándose en cada exploración: el TTO, el DRD, TTF y las aspiraciones. Si en dos exploraciones consecutivas no se detectó aspiración, no se efectuó ningún estudio más de seguimiento.

La cuantificación de los parámetros temporales (TTO, DRD, TTF) se efectuó con el bolo de $5 \mathrm{ml}$ y la viscosidad néctar debido a que se había realizado en estas condiciones en todos los pacientes.

\section{Tratamiento}

En aquellos pacientes en los que videofluoroscópicamente se diagnosticó aspiración se introdujo tratamiento específico, consistente en los casos de aspiración predeglución en el cambio de las características de la dieta (incremento de la viscosidad y reducción del volumen del bolo) y maniobras posturales (flexión cervical para favorecer el cierre de la vía aérea). En los casos de aspiración durante la contracción faríngea el tratamiento consistió en el cambio en las características de la dieta (incrementado la viscosidad y reduciendo el volumen del bolo), maniobras posturales (flexión cervical para favorecer el cierre de la vía aérea) y maniobras activas (deglución su- praglótica para favorecer del cierre de la vía aérea) si el paciente era capaz de colaborar. En los casos de aspiración postdeglución se prescribieron cambios en las características de la dieta (modificando la viscosidad y reduciendo el volumen del bolo) y maniobras activas (maniobra de Mendelson) si el paciente podía colaborar.

Después de cada valoración videofluoroscópica el tratamiento se modificó siguiendo estos mismos principios.

\section{Evaluación de la evolución clínica}

Para evaluar la efectividad de las estrategias de tratamiento para compensar los problemas de seguridad en la deglución se investigó de forma prospectiva la aparición de infecciones respiratorias. La eficacia de la deglución se evaluó analizando el índice de masa corporal (IMC) al ingreso, al mes, y a los 3, 6 y 12 meses de evolución.

La forma de alimentación al año de seguimiento se clasificó en: vía oral normal (el paciente seguía dieta normal); oral modificada (alimentación por vía oral con espesantes para los líquidos); combinación de alimentación oral y por sonda de gastrostomía (el paciente recibía todo el aporte nutricional por boca y los líquidos por la sonda de gastrostomía); y exclusivamente por sonda de gastrostomía.

En nuestro hospital la videofloroscopia es una exploración diagnóstica integrada dentro de la actividad asistencial para evaluar a los pacientes con sospecha clínica de disfagia neurógena. En el momento de su introducción (año 1999) esta exploración fue aceptada por el Comité de Ética del Institut Guttmann.

\section{RESULTADOS}

La puntuación media de afectación de la función cognitiva al ingreso según la escala Rancho los Amigos de Función Cognitiva (RLFC) (17) era de 4 (rango: 2-7), siendo al año de seguimiento de 7 (rango: 6-8) (la respuesta neurológica había mejorado).

La exploración clínica de la deglución mostró una alteración en los reflejos palatal y nauseoso en el $30 \%$ de los pacientes, tos durante la exploración en el $40 \%$ y en ningún paciente se detectó cambios en la calidad de la voz (en el $60 \%$ no era explorable).

Al realizar la primera evaluación el $60 \%$ de los pacientes recibían alimentación por sonda nasogástrica o sonda de gastrostomía, el 10\% combinaban vía oral y sonda de gastrostomía, y el resto seguía alimentación exclusivamente oral.

\section{Exploración videofluoroscópica inicial}

Al analizar la fase oral en la primera exploración se obtuvieron los siguientes resultados: un aumento en el TTO en el 70\% de los casos (TTO medio 3,8 seg.; rango: 
0,8-15 seg.) y alteración en el control lingual en el 60\%: fraccionamiento del bolo durante la deglución en el $60 \%$ y disfunción en el sello glosofaríngeo en el 20\%. En la fase faríngea los hallazgos fueron: TTF medio $0,72 \mathrm{seg}$. (rango: 0,34-1,50 seg.), residuo a nivel de los senos piriformes en el $10 \%$ de los pacientes y alteración en la apertura del EES en un $10 \%$. El tiempo de disparo del reflejo deglutorio (DRD) era de media 0,32 seg. (rango: 0,10$0,80 \mathrm{seg}$.).

$\mathrm{Al}$ analizar el momento de la deglución en el que se produjeron las 10 aspiraciones, 2 eran predeglutorias y 9 se producían durante la contracción faríngea (en un paciente se combinaban dos mecanismos). Sólo en 4 pacientes se desencadenó tos postdeglución de forma que el $60 \%$ eran aspiradores silentes. En 2 pacientes existía penetración al vestíbulo laríngeo.

La consistencia del bolo a la que se producía la aspiración fue: en 2 pacientes con líquido, en 6 con néctar, y en dos con pudin (Fig. 1).

Durante el seguimiento se objetivó una normalización progresiva en la cuantificación de los parámetros que evalúan la función deglutoria, de manera que fue reduciéndose el TTO, TTF y DRD; al igual que también fue disminuyendo el número de pacientes que aspiraban (Fig. 2). Las consistencias en las que se producían las aspiraciones se detallan en la figura 1 .

-Control al mes. El TTO medio era 2,48 seg. (0,34$10)$, el DRD de 0,30 seg. $(0,08-0,80)$ y el TTF de 0,72 seg. $(0,36-1,36)$. Aspiraban 6 pacientes, todos durante la contracción faríngea y 1 además predeglución (combinaba dos mecanismos); el 60\% eran aspiradores silentes.

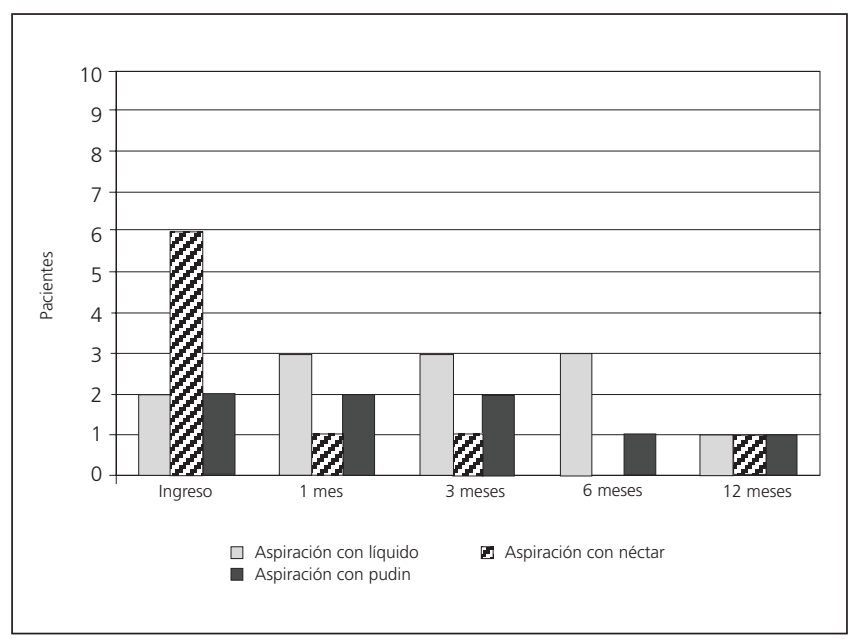

Fig. 1.- Gráfico en el que se detalla el número de pacientes que presentaron aspiración con cada una de las viscosidades utilizadas en la exploración videofluoroscópica. Obsérvese que en la evaluación inicial todos los pacientes aspiran con el líquido, ocho también con el néctar y dos además con el pudin. Al año de seguimiento, de los tres pacientes que siguen aspirando, solamente uno aspira con las tres viscosidades.

-Control a los 3 meses. El TTO medio era de 2,03 seg. (0,2-11), el DRD de 0,29 seg. (0,1-0,8), el TTF de 0,70 seg. $(0,42-1,28)$. Se mantenía el mismo número y momento de las aspiraciones; el 50\% eran aspiradores silentes. En un $20 \%$ existía penetración a vestíbulo laríngeo.

-Control a los 6 meses. El TTO medio fue de 1,32 seg. (0,34-10 seg.), el DRD de 0,25 seg. (0,1-0,8 seg.), el TTF

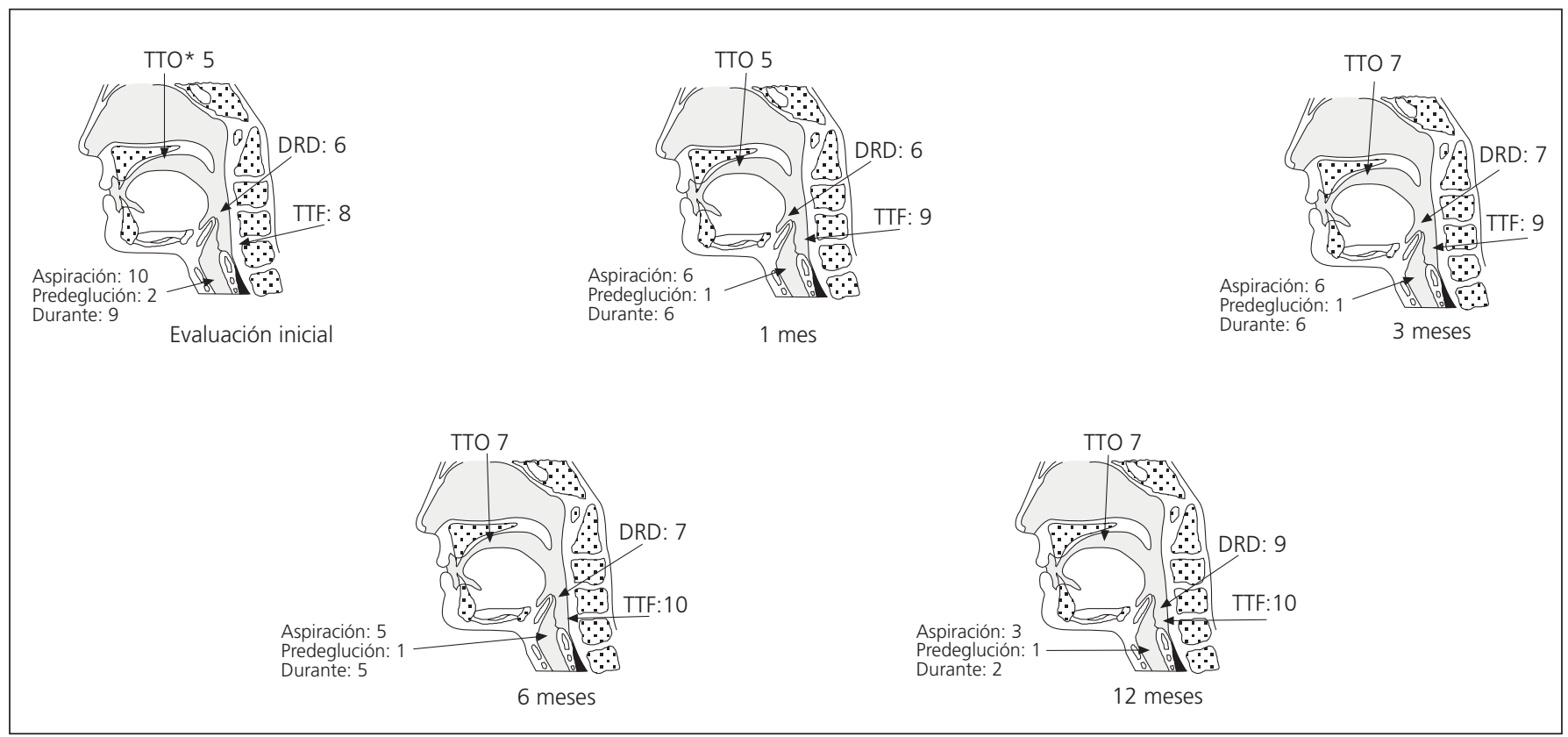

Fig. 2.- Evolución de la cuantificación de los parámetros temporales y de las aspiraciones en las diferentes evaluaciones realizadas. Nótese que al año de evolución únicamente tres pacientes continúan aspirando, se ha normalizado el TTF en todos los casos, el DRD en 9 y el TTO en 7.

*En los ítems (TTO: tiempo de tránsito oral, DRD: tiempo de disparo del reflejo deglutorio, y TTF: tiempo de tránsito faríngeo) se recoge el número de pacientes con una cuantificación dentro de la normalidad. 
de 0,70 seg. (0,5-1,14 seg.). Aspiraban 5 pacientes, todos durante la contracción faríngea y uno predeglución (combinaba dos mecanismos); el 60\% eran aspiradores silentes. En 1 paciente existía penetración a vestíbulo laríngeo.

-Control al año. El TTO medio fue de 1,32 (0,34-10 seg.), el DRD de 0,20 (0,1-0,8 seg.) y el TTF 0,62 (0,41,14 seg.). El número de pacientes en los que se observó aspiración fue disminuyendo, de tal forma que sólo 3 aspiraban (dos durante la contracción faríngea y uno predeglución); el 30\% eran aspiradores silentes. En 1 paciente existía penetración a vestíbulo laríngeo.

\section{Tratamiento}

Después de la primera evaluación se indicaron cambios en las características de la dieta (aumento de la viscosidad del bolo alimenticio) en el $80 \%$ de los pacientes, maniobras posturales (flexión cervical) en el 10\%, y maniobras activas (deglución supraglótica) en el 10\%. El tratamiento se fue modificando en función del resultado obtenido en cada evaluación videofluoroscópica, de manera que los cambios en las características de la dieta se indicaron en el $50 \%$ de los pacientes al mes, tres meses y 6 mes de evolución y en un 30\% al año de seguimiento. Las maniobras posturales y las activas se recomendaron en un $10 \%$ de los pacientes en todos los controles.

\section{Evolución clínica}

Al año de evolución el $80 \%$ de los pacientes seguía alimentación por vía oral (un caso oral modificada y el resto dieta normal) y el 20\% combinaban alimentación por vía oral y sonda de gastrostomía. Durante este periodo ningún paciente presentó infecciones respiratorias. El IMC aumentó durante el seguimiento, siendo al ingreso de 19,3 (rango: 15-22), al mes 19,7 (rango 15-24.9), a los 3 meses 21,3 (rango: 16-26), a los 6 meses 22,3 (19,6-33) y al año de 23,2 (rango: 20,3-33,2).

\section{DISCUSIÓN}

Existen escasos estudios que evalúen las alteraciones de la deglución en pacientes con lesión cerebral traumática. En la literatura la incidencia de disfagia oscila entre el 25 y el $61 \%(2,18,19)$; la variabilidad de los resultados puede ser debida al concepto de disfagia, al método utilizado para su evaluación, a la severidad del TCE y al tiempo de evolución. La mayoría de autores describen la alteración en la eficacia de la fase oral como la disfunción más prevalente en estos pacientes $(2,4,16,18)$.

En un estudio recientemente efectuado en nuestra unidad en pacientes en la fase inicial de un TCE grave detectamos alguna alteración de la fase oral en el $65 \%$ y farín- gea en el $73 \%$. En la fase oral predominaban las alteraciones en la eficacia de la deglución mientras que en la fase faríngea problemas de seguridad, con un $75 \%$ de aspiraciones (20).

En la serie analizada actualmente destaca que a los 6 meses de evolución la mitad de los pacientes no aspiran, y que al año de seguimiento el $70 \%$ de las aspiraciones se han resuelto. La mayoría de las aspiraciones se producen durante la contracción faríngea $(90 \%)$, debido a un retraso en el disparo de la deglución faríngea o a una alteración en la protección de la vía área durante la deglución por un cierre insuficiente de la misma; es de destacar que en el $60 \%$ de estos pacientes existe un retraso en el disparo del reflejo deglutorio. Sólo el $10 \%$ de las aspiraciones son predeglutorias a consecuencia del fallo en el sello glosopalatal de manera que se produce una caída del bolo alimenticio dentro de la faringe antes de que se dispare la deglución faríngea mientras la vía aérea está abierta; en estos casos el TTO está significativamente alargado. A su vez, las aspiraciones postdeglutorias se producen como consecuencia de la existencia de un residuo faríngeo excesivo que se puede aspirar en la siguiente inspiración después de la deglución (en este estudio no se observó ningún de estos casos).

El análisis cuantitativo de la función deglutoria nos permite medir de forma objetiva diferentes parámetros y así definir de forma más precisa la naturaleza del trastorno de la deglución, comparar con otros estudios y evaluar de un modo más exacto su evolución. Este análisis nos ha permitido observar que más de la mitad de los pacientes evaluados tienen un TTO alargado, que se va reduciendo con la evolución de la lesión cerebral y es normal en el $80 \%$ de los pacientes al año de evolución. Además, el retraso en el disparo del reflejo deglutorio existente en la fase inicial también se va normalizando, estando dentro de los límites de la normalidad entre los 6 meses y el año de evolución. El TTF en este grupo de pacientes se encuentra alargado en la valoración inicial en sólo el $20 \%$ de los casos, normalizándose en la totalidad de los pacientes a los 6 meses de evolución. Es de destacar que la mejoría de la función deglutoria va paralela a la mejoría neurológica.

La respuesta fisiológica a la aspiración es la tos refleja, pero en pacientes neurológicos existe un elevado porcentaje de aspiradores silentes, que según la literatura oscila del $40-60 \%(10-13,20)$. En nuestro grupo de pacientes el porcentaje de aspiradores silentes fue del $60 \%$ en la fase inicial de la evolución del TCE habiendo disminuido al año hasta el 30\%. El mecanismo fisiopatológico por el que se producen las aspiraciones silentes no está del todo aclarado aunque estudios recientes sugieren una disfunción en los mecanismos que participan en el reflejo tusígeno: alteración del control neuromuscular por una lesión central o periférica, reducción de la sensibilidad faríngo-laríngea y disminución de los niveles de sustancia $\mathrm{P}(6,21-23)$.

No existe ningún tratamiento específico para la disfagia asociada a los TCE (24), aunque durante los últimos 
años se han descrito diversas técnicas de rehabilitación (dietéticas, posturales, maniobras deglutorias) para compensar las alteraciones biomecánicas $(5,11)$. No obstante, hay muy pocos estudios que demuestren la efectividad de las estrategias terapéuticas para el manejo de la disfagia neurógena en este grupo de pacientes $(12,25,26)$. El tratamiento más adecuado se establece en función del mecanismo fisiopatológico de la aspiración y de la alteración cognitiva conductual. Las estrategias más utilizadas son aquellas que no precisan colaboración por parte del paciente; así, en más de la mitad de los casos nosotros efectuamos cambios en las características de la dieta, seguido de las maniobras posturales, siendo poco utilizadas las maniobras activas que precisaran preservación de la función cognitiva. Lógicamente esto se debe a que el paciente debe comprender cómo se efectúan estas maniobras y, en la práctica, se tienen que excluir un porcentaje importante de pacientes $(12,14)$. Aún así, es importante resaltar que con un manejo terapéutico adecuado la totalidad de estos pacientes podrán seguir, después de un cierto tiempo de evolución, alimentación por vía oral (total o parcial) sin presentar complicaciones respiratorias. Por lo tanto, es básico el diagnóstico y tratamiento correctos de la aspiración durante la fase inicial del TCE.

En conclusión, la presencia de aspiración laringo-traqueal es muy frecuente en pacientes en la fase inicial de un TCE grave, siendo aspiradores silentes más de la mitad de los casos (no detectada clínicamente). La exploración videofluoroscópica nos permite efectuar el diagnóstico del mecanismo fisiopatológico de la aspiración, cuantificar los parámetros deglutorios que determinarán de forma más precisa la evolución de la función orofaríngea, e indicar el tratamiento adecuado a cada paciente, evitándose así las complicaciones respiratorias y mejorando el estado nutricional. Basado en esta experiencia inicial, y a la vista de la evolución de las aspiraciones observada en la exploración videofluoroscópica, aconsejamos efectuar un primer control videofluoroscópico a los 6 meses y un segundo control al año del primero, con el objetivo de someter al paciente a la mínima irradiación y así poder detectar las mejorías evolutivas de una forma más significativa.

\section{BIBLIOGRAFÍA}

1. Winstein CJ. Neurogenic dysphagia. Frequency, progression, and outcome in adults following head injury. Phys Ther 1983; 63(12): 1992-7.

2. Mackay LE, Morgan AS, Bernstein BA. Swallowing disorders in severe brain injury: risk factors affecting return to oral intake. Arch Phys Med Rehabil 1999; 80(4): 365-71.

3. Lazarus C, Logemann JA. Swallowing disorders in closed head trau- ma patients. Arch Phys Med Rehabil 1987; 68(2): 79-84.

4. Field LH, Weiss CJ. Dysphagia with head injury. Brain Inj 1989; 3(1): 19-26.

5. Logemann J. Swallowing disorders caused by neurologic lesions from which some recovery can be anticipated. In: Austin T, editor. Evaluation and treatment of swallowing disorders. Texas; 1998. p. 307-26.

6. Ramsey D, Smithard D, Kalra L. Silent aspiration: what do we know? Dysphagia 2005; 20(3): 218-25.

7. Morgan A, Ward E, Murdoch B, Bilbie K. Acute characteristics of pediatric dysphagia subsequent to traumatic brain injury: videofluoroscopic assessment. J Head Trauma Rehabil 2002; 17(3): 220-41.

8. Leder SB. Fiberoptic endoscopic evaluation of swallowing in patients with acute traumatic brain injury. J Head Trauma Rehabil 1999; 14(5): 448-53.

9. Tolep K, Getch CL, Criner GJ. Swallowing dysfunction in patients receiving prolonged mechanical ventilation. Chest 1996; 109(1): 16772.

10. Splaingard ML HB, Sulton LD, Chaudhuri G. Aspiration in rehabilitation patients: videofluoroscopy vs bedside clinical assessment. Arch Phys Med Rehabil 1988; 69(8): 637-40.

11. Pouderoux P, Kahrilas PJ. Deglutitive tongue force modulation by volition, volume, and viscosity in humans. Gastroenterology 1995; 108(5): 1418-26.

12. Spechler SJ. AGA technical review on treatment of patients with dysphagia caused by benign disorders of the distal esophagus. Gastroenterology 1999; 117(1): 233-54.

13. Terré R, Mearin F. Oropharyngeal dysphagia after the acute phase of stroke: predictors of aspiration. Neurogastroenterol Motil 2006; 18(3): 200-5.

14. Schurr MJ, Ebner KA, Maser AL, Sperling KB, Helgerson RB, Harms B. Formal swallowing evaluation and therapy after traumatic brain injury improves dysphagia outcomes. J Trauma 1999; 46(5): 817-21; discussion 821-3.

15. Morgan A, Ward E, Murdoch B. Clinical progression and outcome of dysphagia following paediatric traumatic brain injury: a prospective study. Brain Inj 2004; 18(4): 359-76.

16. Mackay LE, Morgan AS, Bernstein BA. Factors affecting oral feeding with severe traumatic brain injury. J Head Trauma Rehabil 1999; 14(5): 435-47.

17. Hagen C. Language disorders secondary to closed head injury: diagnosis and treatment. Top Lang Disord 1981; 1: 73-87.

18. Cherney LR, Halper AS. Swallowing problems in adults with traumatic brain injury. Semin Neurol 1996; 16(4): 349-53.

19. Morgan A, Ward E, Murdoch B. Clinical characteristics of acute dysphagia in pediatric patients following traumatic brain injury. $\mathbf{J}$ Head Trauma Rehabil 2004; 19(3): 226-40.

20. Terre R, Mearin F. Oropharyngeal dysphagia after severe traumatic brain injury: predictor of dysphagia (en prensa).

21. Arai T, Yasuda Y, Takaya T, Toshima S, Kashiki Y, Yoshimi N, et al. ACE inhibitors and symptomless dysphagia. Lancet 1998; 352(9122): 115-6.

22. Arai T, Yoshimi N, Fujiwara H, Sekizawa K. Serum substance P concentrations and silent aspiration in elderly patients with stroke. Neurology 2003; 61(11): 1625-6.

23. Arai T, Sekizawa K, Yoshimi N, Toshima S, Fujiwara H. Cabergoline and silent aspiration in elderly patients with stroke. J Am Geriatr Soc 2003; 51(12): 1815-6.

24. Logemann JA PJ, Mackay LA. Disorders of nutrition and swallowing: intervention strategies in the trauma centre. J Head Trauma Rehabil 1994; 9(1): 43-56.

25. DePippo KL, Holas MA, Reding MJ, Mandel FS, Lesser ML. Dysphagia therapy following stroke: a controlled trial. Neurology 1994; 44(9): 1655-60.

26. Neumann S, Bartolome G, Buchholz D, Prosiegel M. Swallowing therapy of neurologic patients: correlation of outcome with pretreatment variables and therapeutic methods. Dysphagia 1995; 10(1): 1-5. 